\title{
Género y conflictos socioambientales: Una experiencia de investigación-acción participativa con mujeres dirigentes
}

\author{
[Gender and environmental conflicts: An experience of participatory action-
} research with female grassroots leaders]

\author{
Antonia Zambra Álvarez \& Evelyn Arriagada Oyarzún \\ Universidad Diego Portales
}

\begin{abstract}
Resumen
A partir de las contribuciones teóricas y metodológicas de la ecología política feminista y la investigación-acción participativa, este artículo ilustra cómo la experiencia de mujeres en torno a problemas de contaminación, acceso y/o uso a los recursos naturales puede gatillar procesos de politización y acción colectiva. Al respecto, las posibilidades y limitaciones que emergen de la experiencia de ser mujer y ser dirigente juegan un rol central para el abordaje de los problemas socioambientales en Chile. Tomando como caso una iniciativa piloto de formación de mujeres dirigentes de zonas rurales de la Región Metropolitana (Chile), se buscó relevar la perspectiva de género en la formación e investigación en torno a la relación sociedadnaturaleza. Los resultados muestran que las participantes ejercen una forma particular de liderazgo, donde el cuidado de la familia, la comunidad y el territorio constituyen los principales ejes de movilización social y formas cotidianas de hacer política.
\end{abstract}

Palabras clave: ecología política feminista, investigación acción participativa, género, medio ambiente.

\begin{abstract}
Based on theoretical and methodological contributions from the feminist political ecology approach, and the participatory action-research perspective, this article seeks to illustrate that the experience of women around pollution, access and use of natural resources can trigger processes of politicization and collective action. In this regard, the possibilities and limitations related to being women and grassroots leaders, play a central role in dealing with environmental problems in Chile. Our case focuses on a pilot project of training, targeted to female grassroots leaders, from rural areas of the Metropolitan Region of Santiago (Chile). In this experience, we sought to highlight the incorporation of a gender perspective, both in teaching and researching processes, around the relationship between nature and society. Preliminary results show that participants deploy a form of leadership in which care of the family, the community and the territory constitute the main axes of social mobilization and doing everyday politics.
\end{abstract}

Keywords: feminist political ecology, participatory action-research, gender, environment.

Contacto: La comunicación sobre este artículo debe ser enviada a Antonia Zambra Álvarez, email: antonia.zambra@mail.udp.cl 


\section{INTRODUCCIÓN}

La incorporación del enfoque de género para abordar los problemas socioambientales recientes se ha debido en parte importante a las contribuciones teóricas de una ecología política feminista en construcción (Bolados, 2017). Tomando en consideración una amplia gama de perspectivas sobre género y medio ambiente, este programa de investigación - que a su vez es una práctica política (Bustos, Prieto \& Barton, 2015) - plantea la importancia de mirar la manera como significamos, habitamos y transformamos el medio ambiente a través del género, comprendiendo el rol que juegan en ello las relaciones de poder involucradas en las dinámicas de acceso y control de los recursos (Hawkins et al., 2011).

A partir de las contribuciones del ecofeminismo en sus diversas propuestas (e.g., Agarwal, 1992; Merchant, 1980; Plumwood, 1993; Shiva \& Mies, 1993), los estudios feministas en torno a la producción de conocimiento (Haraway, 1991; Harding, 1986), los estudios sobre dinámicas de género en acceso y control de los recursos (Harris, 2006, 2015, 2017; Sultana, 2011), las críticas feministas al desarrollo (Carney, 1992), los estudios feministas poscoloniales o los postulados posestructuralistas en torno a la producción de subjetividades de género mediadas por el entorno (Nightingale, 2006; Sundberg, 2017), la ecología política feminista se nutre de una agenda de investigación flexible y heterogénea.
Uno de los principales puntos de convergencia y línea argumentativa para la ecología política feminista, radica en el reconocimiento que los roles $\mathrm{y}$ diferencias de género social $\mathrm{y}$ biológicamente construidos, se dan desde y a través de los vínculos e intercambios que las sociedades establecen con su vida ecológica material (Sundberg, 2017). Dichas relaciones, están atravesadas por dinámicas de poder y políticas de desarrollo que tomarán expresiones concretas en las particularidades territoriales, a distintas escalas de análisis. Adicionalmente, desde un punto de vista epistemológico y metodológico, la ecología política feminista ha cuestionado la manera colonial, cientificista y patriarcal que ha predominado en la construcción de conocimiento sobre el medioambiente $\mathrm{y}$ ha intentado formas innovadoras y más horizontales de investigación y acción (Sundberg, 2017). En tal sentido, es posible vislumbrar múltiples posibilidades de convergencia entre este enfoque y los métodos y técnicas participativas de investigación, especialmente fructíferos en nuestra región. Al respecto, un aporte central de este trabajo se constituye a partir de la incorporación de la investigación acción participativa (en adelante IAP) como elemento movilizador de una co- $^{-}$ construcción de conocimiento sobre género y medioambiente.

En Chile, si bien aún son pocos los estudios e iniciativas que abordan los conflictos socioambientales desde el lente de la ecología 
política feminista (Arriagada, 2019; Bolados, 2017); es conveniente señalar que se trata de un enfoque que está siendo incorporado de manera gradual en el debate académico y en paralelo a la constatación de un protagonismo femenino en las luchas socioambientales a nivel nacional y continental. El caso emblemático de la constitución de la organización de Mujeres de Zonas de Sacrificio en Resistencia de Quintero y Puchuncaví en 2016, da cuenta de cómo estos procesos alternativos de movilización y acción colectiva en Chile han permitido levantar denuncias en torno a la violación de derechos humanos fundamentales (derecho a la salud, a vivir en un medio ambiente libre de contaminación, reproductivos), así como también, reinterpretar los efectos del extractivismo y el patriarcado en territorios afectados por contaminación y/o escasez de recursos (Bolados, 2017).

En este marco reflexivo, el presente artículo busca contribuir al cuerpo de trabajo de la ecología política feminista en Chile, a partir de la utilización de este enfoque en una iniciativa piloto de formación de mujeres dirigentes provenientes de zonas rurales de la Región Metropolitana de Santiago. Planteada como un ejercicio de diálogo, acompañamiento y coconstrucción de conocimiento ambiental para la acción desde una perspectiva de género, esta iniciativa toma como inspiración teórica los planteamientos de la ecología política feminista y, como inspiración metodológica, los principios, métodos, técnicas recursos de la IAP en el abordaje de problemas socioambientales recientes en Chile. En tal sentido, las preguntas que originaron y sustentaron este trabajo fueron dos, a saber, ¿cómo las mujeres rurales de la Región Metropolitana experimentan las intervenciones y transformaciones ambientales en sus territorios? y, ¿cómo -desde una alianza entre academia, instituciones y comunidadespodemos producir conocimiento para la acción ambiental, de forma más horizontal y participativa?

Así, entre junio y septiembre de 2018 -período álgido de las movilizaciones feministas que remecieron al país- en un trabajo conjunto entre el Observatorio de Desigualdades (Universidad Diego Portales), la Fundación para la Superación de la Pobreza de la Región Metropolitana (FUSUPO) y el Centro de Estudios del Conflicto y la Cohesión Social (COES), la iniciativa escuela de mujeres dirigentes en temáticas socioambientales y territoriales tuvo como objetivo principal fortalecer habilidades de mujeres dirigentes que se encuentran enfrentando algún tipo de problemática ambiental en sus territorios. Entre estas problemáticas destacan la desaparición gradual de actividades agrícolas, debido a la escasez de agua en pozos y norias, o problemas a la salud por contaminación de aire, agua o suelo, por nombrar algunos.

La escuela se articuló en torno a cuatro sesiones taller. A partir de un ejercicio participativo de identificación de experiencias de género vinculadas a las problemáticas socioambientales específicas de cada localidad, los resultados muestran que las las dirigentes ejercen una forma particular de liderazgo donde el cuidado de la familia, la comunidad y el territorio 
constituyen ejes de movilización social y formas cotidianas de hacer política.

Se concluye que los elementos teóricos que otorga la Ecología Política Feminista y la aproximación metodológica que brinda la IAP, resultan especialmente relevantes para articular y movilizar una reflexión abierta alrededor de las múltiples experiencias de género en su relación con la naturaleza, y particularmente, como variable clave para problematizar en torno a las causas y efectos de los conflictos socioambientales en Chile. Así, el presente trabajo ilustra un ejercicio de experimentación, donde academia, instituciones y comunidades convergen para construir conjuntamente otros tipos de conocimiento sobre lo ambiental, que incorporan activamente el punto de vista de las mujeres protagonistas y desafían los constructos teóricos, epistemológicos y metodológicos dominantes hoy en estas temáticas.

Género y medioambiente en los estudios sociológicos

Si bien los estudios feministas y de género han sido fundamentales para el desarrollo contemporáneo de la sociología (Güereca, 2016), las teorizaciones en torno a estas temáticas han sido escasas en la sociología ambiental. De acuerdo a lo planteado por Kennedy y Dzialo (2015), este subcampo disciplinar ha tendido a considerar el género como una variable sociodemográfica más y se ha centrado en acumular evidencia -especialmente cuantitativa- sobre el involucramiento y participación de mujeres en materias ambientales, especialmente en países desarrollados. De acuerdo con esta autora, uno de los principales consensos en la sociología ambiental sería que las mujeres tienden a expresar una mayor preocupación y apoyo a la protección ambiental, aunque no hay hipótesis, conceptos ni teorías sobre los mecanismos causales que explican dicha propensión diferenciada en torno al género. Tampoco existirían dichos insumos, dicen Kennedy y Dzialo (2015), para comprender lo que esas diferencias significan para hombres y mujeres.

A pesar de no constituirse en un corpus propio dentro la sociología ambiental, la relación entre género $\mathrm{y}$ activismo ambiental ha sido problematizada por algunas sociólogas norteamericanas, especialmente en el entrecruce de campos como la justicia ambiental y los estudios feministas. Así, por ejemplo, Krauss (2008) se pregunta por las características del activismo ambiental de mujeres blancas de clase baja en Estados Unidos. Su investigación muestra la importancia de las creencias subjetivas y las experiencias cotidianas, especialmente en torno a la maternidad y los roles tradicionales de género, como un elemento constitutivo de dicha politización. Por su parte, Bell y Braun (2010), llaman la atención sobre la masiva presencia de mujeres en organizaciones ambientales que buscan la justicia ambiental, y describen cómo las identidades de género permean este activismo de forma diferenciada en hombres y mujeres Apalache. Es interesante observar, al respecto, que la preponderancia de la maternidad aparece también aquí como factor identitario que gatilla la politización femenina 
en conflictos ambientales, especialmente en aquellos que comprometen la salud de los hijos e hijas y otras personas dependientes.

Para el caso chileno, el estudio de las 14 dirigentes de Arica realizado por Arriagada (2019) muestra que las dinámicas de género no solo son relevantes para comprender el activismo de las mujeres, sino también la construcción de las experiencias cotidianas en torno a la contaminación. Basada en los planteamientos de Krauss (2008) y Kaplan (1997), Arriagada (2019) relee la politización de la maternidad en su relación con el sufrimiento ambiental (Auyero \& Swistun, 2008). Considerando cómo la posición de clase interactúa con la dominación de género en disputas en torno al conocimiento y los cuidados, la autora concluye que no se puede comprender dicha politización sin entender las experiencias feminizadas de sufrimiento ambiental.

Si bien estos estudios permiten aproximarse a algunas de las interrogantes planteadas por Kennedy y Dzialo (2015) desde la sociología ambiental, éstos no son suficientes para construir una mirada comprehensiva sobre las relaciones entre género $\mathrm{y}$ medioambiente. A pesar de que se recurre a conceptualizaciones que se hacen tanto desde la estructura como desde la agencia, la mirada sociológica no incorpora la relación entre las múltiples escalas en que se producen y reproducen las relaciones de género, ni de la manera en que el género mismo puede ser redefinido, a través de las interacciones entre humanos y naturaleza (Harris, 2017). Es preciso, por tanto, recurrir a perspectivas que rebasen los aportes de una sola disciplina y que permitan comprender la relación recursiva entre género y medioambiente en toda su magnitud. Tal es el caso de la ecología política feminista.

Género y medioambiente: Más allá de la sociología

Ampliar la mirada hacia la relación género y medio ambiente, más allá de la disciplina sociológica, implica remontarnos a los primeros intentos de académicas y activistas, durante la década de 1970, de incorporar los objetivos y la teoría feminista a los estudios del desarrollo. Para dar contenido a estos programas de planificación e intervención dirigidos hacia los países del sur, un soporte teórico y conceptual asociado a las primeras corrientes ecofeministas de la época (Merchant, 1980; Shiva \& Mies, 1993) constituirá el punto de partida de un amplio repertorio de perspectivas y abordajes en torno a esta temática que, en sus encuentros $\mathrm{y}$ desencuentros, darán forma al cuerpo de trabajo de una ecología política feminista que hasta hoy sigue siendo materia de debate (Bolados, 2017; Hawkins et al., 2011).

Se trata de contribuciones que irán en paralelo al surgimiento de la ecología política como respuesta intelectual para explicar el contexto de transformación económica, política, social y cultural de la segunda mitad del siglo XX. Durante este período, la ecología (apolítica) tradicional se vuelve un referente insuficiente para comprender y brindar soluciones a los problemas socio ambientales contemporáneos y es necesario interpelarla desde una mirada 

experiencia de investigación-acción participativa con mujeres dirigentes. Revista de Sociología, 34(1), 147-165. doi: 10.5354/0719-529X.2019.54270

crítica que permita constituir una alternativa de pensamiento renovado sobre la naturaleza (Leff, 2006). Utilizando como base intelectual corrientes tan diversas como el legado marxista de la ecología, las propuestas posestructuralistas/posthumanistas o los estudios poscoloniales, la ecología política interpelará un presente en el cual los seres humanos han conseguido convertir la naturaleza en un recurso que puede ser explotado e intercambiado como objeto de mercancía. Desde un lente latinoamericanista, autores tales como Leff (2006), Escobar (2011) y Alimonda (2006, 2011), llevarán esta reflexión crítica a la situación de nuestro continente en tanto receptáculo de cargas ambientales y fuente de recursos de una economía extractivista que es, a su vez, herencia de una historia ambiental de colonización.

De este marco reflexivo, se desprende y apoya la ecología política feminista para sostener que existen consecuencias tanto materiales como simbólicas en la relación género y medio ambiente (Hawkins et al., 2011). A partir de esta afirmación, la ecología política feminista no solo desafiará aquellos preceptos iniciales del ecofeminismo, que sostienen una diferencia intrínseca en la relación género y medio ambiente (Ojeda, 2011; Sundberg, 2017), sino que planteará la importancia de la dimensión histórica y social en el proceso de constitución del género en tanto éste es construido en y a través de la naturaleza.

Específicamente respecto del legado del materialismo histórico en la ecología política feminista, el foco de investigación estará puesto en las formas capitalistas de construcción y producción de la naturaleza, así como en las dinámicas de género involucradas en los procesos de despojo, acceso y uso de los recursos (Elmhirst, 2018). Destaca con relación a este argumento, cómo variables de género, clase, raza y etnia permiten explicar las transformaciones ambientales recientes, en tanto se reconoce que los procesos e interacciones que los seres humanos llegan a establecer con el entorno inmediato se producen de manera desigual, mediadas por relaciones de poder a distintas escalas y niveles de la esfera social. Así, aquello que sería esperado por una persona en base al género que le es socialmente asignado, permitiría en parte explicar lo que una persona llega a conocer sobre el medio ambiente, qué o cuánto acceso y control se tiene sobre los sistemas ecológicos, qué formas adoptan las resistencias a los riesgos y amenazas del entorno, o bien, qué razones movilizan la acción política y social en contextos de problemas o crisis medio ambientales (Robbins, 2012; Rocheleau, Thomas-Slayter \& Wangari, 2004). Los estudios posestructuralistas, por otro lado, se centrarán en la conformación de aquellas identidades sociales forjadas en y a través de la relación con el medio ambiente en su intercambio cotidiano (Sundberg, 2017). Se trata de un programa de investigación, en este caso, que nos permite transitar de manera flexible desde el espacio cotidiano del cuerpo, la vida doméstica y las comunidades hacia la producción de discursos y prácticas de género asociadas a políticas y procesos nacionales e internacionales globales (Elmhirst, 2018). Como plantea 
Hawkins et al. (2011), poder navegar entre escalas de análisis nos brinda la posibilidad de comprender las problemáticas socioambientales a partir de la escala íntima del cuerpo como espacio de negociación. En otras palabras, "el cuerpo es un sitio donde los temas ambientales se materializan" (Hawkins et al., 2011, p. 244).

Los estudios feministas sobre la ciencia y los estudios poscoloniales, por su parte, nos invitarán a identificar aquel conocimiento ambiental situado y muchas veces invisibilizado, en contraste con el privilegio epistémico de formas (masculinas, científicas) predominantes de producción de conocimiento. Tomando la crítica feminista de la ciencia, la Ecología Política Feminista cuestionará la forma como el conocimiento ambiental es producido, así como también, qué y quiénes cuentan como productores de este conocimiento (Haraway, 1991; Harding, 1986; Haraway, 1991). Del mismo modo, el pensamiento decolonial, intentará ir un poco más allá, al plantear la necesidad de reconocer y repensar el lenguaje conceptual que emerge desde América Latina, África, el mundo indígena o los espacios marginalizados de la academia, con el fin de proponer nuevas categorías que desafíen los preceptos universalizantes del conocimiento occidental (Elmhirst, 2018).

En todas estas propuestas teóricas -que presentan diferencias y/o discrepancias- lo que se persigue es reconocer que las desigualdades seguirán reproduciéndose mientras el conocimiento, las prácticas y el activismo ambiental de las mujeres continúe siendo invisibilizado. En este sentido, la ecología política feminista busca constituir marcos éticos alternativos para imaginar una relación naturaleza-sociedad basada en conceptos de relacionalidad, cuidado o responsabilidad y que nos permitan, a su vez, desafiar las actuales formas neoliberales de desarrollo basadas en el consumo y la extracción permanente de los recursos naturales (Elmhirst, 2018; Sundberg, 2017).

Tomando en consideración las reflexiones preliminares de este apartado, las orientaciones actuales en la ecología política feminista apuntan a superar el foco inicialmente puesto en las vulnerabilidades de las mujeres, y su relación con fuerzas estructurales y ambientales más amplias, característico de las primeras etapas del ecofeminismo. En contraste, se pone el acento en las posibilidades de agencia, especialmente considerando que el conocimiento femenino es crucial para comprender la degradación ambiental y para enfrentar los desafíos actuales y futuros en torno a ellos (Buechler \& Hanson, 2015).

En esta línea, y como se abordará en el siguiente apartado, nuestro trabajo busca contribuir a la construcción de una ecología política feminista en Chile (Bolados, 2017), y el sur global, donde converjan los elementos aquí planteados con elementos epistemológicos y metodológicos de la tradición sociológica latinoamericana, como es el caso de la IAP. 
Re-explorando horizontes epistemológicos y metodológicos: la Investigación Acción Participativa (IAP)

La problematización sobre conflictos socio ambientales desde una perspectiva de género requiere replantearse necesariamente las formas y los mecanismos a través de los cuales el conocimiento ambiental es producido y legitimado en los distintos espacios de producción de saberes. Se trata de reconocer, en este caso, que existen ciertas presunciones teóricas, conceptuales y metodológicas a las que se debe prestar especial atención cuando lo que buscamos es explorar e identificar aquella experiencia ambiental invisibilizada que cruza la vida de las mujeres (Sundberg, 2017). En este sentido, parte de los objetivos de trabajo de la ecología política feminista buscan cuestionar la conceptualización de los sujetos y categorías que convencionalmente hemos utilizado para estudiar la relación naturaleza-sociedad ¿Qué escala de la vida ecológica resulta relevante para analizar esta interacción desde una perspectiva de género? ¿Qué subjetividades se nos revelan cuando intentamos dejar de lado aquellas nociones de género pre-establecidas?

Desde esta perspectiva, la ecología política feminista plantea la importancia de la intimidad y la vida cotidiana como escalas de análisis, así como la apertura a reconocer la multiplicidad de identidades concebidas al alero de los saberes y prácticas ambientales que se desenvuelven en esta cotidianeidad (Butler, 1999; Sundberg, 2017). Para reconocer aquella experiencia ambiental que ha sido de alguna manera marginada y/o invisibilizada, la ecología política feminista señala la necesidad de incorporar cierta creatividad metodológica a los objetivos de trabajo e investigación, ya sea a partir del desarrollo de métodos mixtos o bien, desde un enfoque exclusivamente cualitativo que se aproxime desde una mirada sensible a las experiencias, categorías y espacios de los sujetos investigados.

En este marco reflexivo, nos interesa rescatar los aportes de la IAP, como horizonte epistemológico y metodológico para el abordaje crítico de los conflictos socioambientales desde una perspectiva de género.

La IAP, cuyo cuerpo de trabajo se ha venido desarrollando desde la década de 1970 sostiene, a grandes rasgos, que la producción de conocimiento debe estar orientada y comprometida con la transformación y la acción social (Fals Borda, 2009). Conseguir este propósito solo será posible en el ejercicio activo de vincular teoría y práctica a partir de la reflexión e interacción de todos y todas las participantes del proceso investigativo. Se trata, en este sentido, de un giro epistemológico que busca situar al grupo en un enfoque colaborativo en el cual un dispositivo metodológico articula tanto los intereses de investigación como los de formación (Arevalo et al., 2016). A su vez, reconoce la necesidad de explorar nuevas metodologías con el doble propósito de innovar la forma como hacemos investigación, al mismo tiempo que movilizar a los sujetos para la acción. De esta manera, la IAP buscará establecer una relación más horizontal entre investigador y sujeto investigado a partir de una mayor 
consciencia y compromiso por parte del investigador respecto de su rol y posición en las problemáticas y/o conflictos que investiga, así como también un mayor involucramiento e incidencia del sujeto investigado en el proceso de investigación. Para alcanzar estos objetivos la IAP requiere por parte de sus participantes, la apertura hacia lógicas alternativas de enseñanza y aprendizaje que posibiliten la construcción de nuevas relaciones entre los sujetos involucrados en el proceso. En este sentido, la flexibilidad orientada a repensar de manera constante las temáticas a abordar, así como el método y herramientas de trabajo, constituye elementos clave para incorporar las subjetividades de los participantes y al mismo tiempo diluir los roles de poder que puedan emerger en el espacio de trabajo (Cortés, Díaz, Flores \& Monsalves, 2016).

\section{MÉTODO}

En un trabajo conjunto entre la FUSUPO, el Observatorio de Desigualdades de la Universidad Diego Portales y el COES, la iniciativa "Escuela de dirigentas en temáticas socio-ambientales y territoriales", desarrollada entre junio y octubre de 2018, se propuso fortalecer habilidades dirigenciales de mujeres provenientes de organizaciones de zonas rurales de la Región Metropolitana que se encuentran enfrentando algún tipo de conflicto socioambiental en sus respectivos territorios. Basándose en el diálogo entre conocimiento académico, institucional y comunitario, esta propuesta se planteó como un espacio piloto de experimentación e intercambio de herramientas teóricas y prácticas para la investigación y la acción colectiva en torno a problemáticas socioambientales existentes en la región.

La Escuela contó con un total de 45 asistentes, de los cuales 35 fueron mujeres dirigentes pertenecientes a las comunas de Til-Til, María Pinto, Alhúe y San Pedro de Melipilla, todas localidades rurales de la Región Metropolitana donde el programa Servicio País de la FUSUPO llevaba un período importante realizando intervenciones a nivel local en miras a conectar, activar y visibilizar a las comunidades y sus recursos a partir de las oportunidades contextuales del territorio y teniendo como horizonte la superación de la pobreza y la exclusión (Servicio País, 2019). Estas mujeres pertenecían a un amplio abanico de organizaciones territoriales, funcionales o ambientales (como juntas de vecinos, comités ambientales comunales, o centros de padres y apoderados, entre otros). La selección de las participantes se hizo por medio de la FUSUPO, a través de los profesionales del programa Servicio País que trabajaban en los territorios mencionados. Debido al foco de la FUSUPO, la invitación tenía como grupo objetivo a mujeres de grupos socioeconómicos más vulnerables que trabajaran actualmente o pudieran comenzar a trabajar con los profesionales de Servicio País, sin distinción etaria, ni de pertenencia organizacional. Además de estos elementos, 
quienes participaron de la iniciativa eran en su mayoría madres o cuidadoras de personas dependientes, cuyo rango de edad oscilaba entre los 35 y los 85 años y habían nacido o llevaban más de 20 años viviendo en las comunas mencionadas.

Desde el punto de vista académico, la iniciativa apuntaba a dos objetivos centrales. El primero, basado en los planteamientos teóricos de la ecología política feminista, buscaba comprender cómo las mujeres rurales de la Región Metropolitana estaban experimentando las intervenciones y transformaciones ambientales en sus territorios. Ello implicaba no sólo saber si sus vivencias $\mathrm{y}$ formas de afrontar las problemáticas ambientales eran diferenciadas por género, sino también relevar los marcos interpretativos que ellas mismas usaban para hacer alusión a ello (y que significaba no imponerles categorías pre-construidas en torno a la relación género-medioambiente). Por otra parte, tomando los principios inspiradores de la IAP, se buscaba experimentar una forma más horizontal y participativa de construcción de conocimiento para la acción. En este sentido, si bien existían ciertas ideas teóricas o metodológicas que inspiraban la iniciativa, la forma de abordar los contenidos y las metodologías específicas para cada sesión serían definidos y negociados en conjunto con las participantes, y durante el desarrollo de la iniciativa.
¿Cómo emerge la dimensión género y medio ambiente en el proceso de gestación de la Escuela?

Para comprender el primer objetivo, es importante preguntarse ¿cómo emerge el género y su relación con los conflictos socioambientales en este proceso de gestación de la Escuela? Existen dos antecedentes relevantes que explican la incorporación de la perspectiva de género $\mathrm{y}$ medio ambiente en este intercambio entre instituciones. En primer lugar, venía desarrollándose hace un tiempo dentro del equipo regional de la FUSUPO la idea de fortalecer habilidades dirigenciales en el marco de programa de intervención Servicio País. En este ejercicio de identificar y catastrar líderes y lideresas presentes en los territorios, los profesionales Servicio País habían ido constatando que en la mayoría de los casos la responsabilidad dirigencial (juntas de vecinos, asociación de adultos mayores, organizaciones sociales) era asumida por mujeres. En segundo lugar, como equipo del Observatorio de Desigualdades, veníamos desarrollando, a partir de un proceso reflexivo desde la ecología política feminista, la importancia de incorporar la dimensión de género para comprender los procesos de feminización que estaban adquiriendo las organizaciones sociales medioambientales tanto en Chile como en América Latina durante las últimas décadas. Alrededor de estos antecedentes surge la idea de experimentar en torno a la posibilidad de desarrollar una experiencia formativa y de intercambio de saberes que tuviese como foco 
exclusivamente a mujeres. A partir de las distintas instancias de conversación, intuíamos, por ejemplo, que las mujeres deberían contar con un espacio para hablar y reflexionar sobre su experiencia dirigencial y que esta experiencia podría ser muy diferente a aquella vivenciada desde formas masculinas de liderazgo. Sospechábamos, además, que ese "algo" que tenían que contar no había sido necesariamente verbalizado por ellas mismas, en tanto se trataba de aquel conocimiento situado y localizado en los márgenes, invisibilizado, postergado. Finalmente sabíamos que la única posibilidad de relevar aquel saber ambiental y territorial particular era generar un espacio seguro $y$ protegido, donde ellas tendrían la posibilidad de expresarse sin miedo y sin cuestionamientos. De ahí emerge la idea de desarrollar una instancia de formación e intercambio de saberes a partir de talleres orientados hacia mujeres dirigentes de comunas rurales de la Región Metropolitana, donde el programa Servicio País se encontraba trabajando.

¿Cómo producir conocimiento para la acción ambiental de forma más horizontal y participativa?

Respecto al segundo objetivo, tomamos los principios inspiradores, la metodología y varias técnicas y recursos de la IAP para hacer emerger el conocimiento sobre los distintos ejes temáticos de la escuela. Así, por ejemplo, cabe mencionar que la dimensión ambiental como foco de las problemáticas territoriales locales, se hizo presente en la primera sesión de la Escuela sin haberla traído forzosamente.

En la Imagen $\mathrm{N}^{\circ} 1$ se observa una de las líneas de tiempo elaborada por un grupo de mujeres dirigentes de la localidad de San Pedro de Melipilla. La línea de tiempo es una herramienta de recolección de información sobre la cual las participantes van dibujando y escribiendo en un papelógrafo - de manera consensuada - los acontecimientos que el grupo considera más destacables de los últimos años (Red Cimas, 2009). Para este ejercicio en particular, se le pidió a cada grupo (conformado por localidad) que trabajara la línea de tiempo en función de alguna problemática vivenciada en sus territorios que les pareciera relevante. Las participantes debían situar esta problemática en el contexto temporal en el que se había desarrollado, además de reconocer las causas y efectos de esta situación en la población y sus habitantes.

A partir de esta pregunta abierta, todos los grupos identificarían problemas de afectación al medio ambiente tales como escasez de agua, contaminación de aire, agua y suelo, consecuencias negativas a la salud, gradual desaparición de actividades tradicionales.

Las sesiones de la Escuela terminaron articulándose alrededor de tres grandes ejes temáticos: actores sociales $\mathrm{y}$ conflictos territoriales (junio), género y medio ambiente (julio) y emociones y acción colectiva (agosto), cuyos resultados fueron recogidos a partir de un registro etnográfico de audio y toma de notas de campo durante cada uno de los talleres. Para ello, las participantes firmaron un 
consentimiento informado al inicio de la escuela, en la cual autorizaban el uso de esos audios e imágenes para fines institucionales y académicos de difusión de la iniciativa.

Figura 1. Línea de tiempo participativa sobre escasez del recurso hídrico en la comuna de San Pedro de Melipilla elaborada por las participantes de la escuela.

La primera sesión implicó poner a prueba nuestro primer diseño metodológico, que dividía la jornada en una parte expositiva (de un/a académico/a experto/a) y una parte de taller con el uso de recursos de la IAP. Durante la segunda parte de esta primera sesión, no solo emergió la problemática ambiental como la más relevante sino también se evidenció que había que repensar la metodología de las sesiones para profundizar el componente participativo. De este modo, para la segunda sesión y las restantes, los recursos de la IAP pasaron a ser los elementos dinamizadores de un conocimiento que se creaba in situ. En tal sentido, las sesiones consistían mayoritariamente en talleres (mapas corporales y emocionales, juego de roles, entre otros) que relevaban las interpretaciones, significados $\mathrm{y}$ contenidos que las participantes asignaban a cada uno de los ejes temáticos. El rol de académicos y académicas también cambió, pasando de una lógica expositiva hacia una orientada a escuchar, acompañar y retroalimentar los elementos que surgían en los talleres.

Cada versión de los talleres se realizó en una sala de la Facultad de Psicología de la Universidad 
Diego Portales, llamada sala Terapia, cuyo mobiliario permitía la flexibilidad suficiente para combinar distintos tipos de actividades, en una misma sesión. El uso de este espacio y la idea del formato taller como método del curso, buscaron justamente propiciar un espacio de reflexión colectiva y relevar la importancia del aprehender-haciendo como estrategia pedagógica.

Los hallazgos que se recogen a continuación sintetizan algunas de las reflexiones colectivas que emergieron durante las sesiones y en las instancias de evaluación posterior de la iniciativa, en torno a la importancia del cuidado como agente canalizador y gatillador de acción colectiva que emana de la experiencia feminizada de dirigencia frente a problemáticas socioambientales. Estas reflexiones fueron reexaminadas tras la implementación de la Escuela, para hacerlas dialogar con ideas, conceptos e indagaciones empíricas de la ecología política feminista.

\section{RESULTADOS}

A partir de un trabajo desde la IAP con mujeres dirigentes de zonas rurales de la región Metropolitana en torno a la experiencia de ser mujer y ser dirigente en un territorio degradado o contaminado como es el caso de Tiltil, San Pedro de Melipilla, Alhué y María Pinto (Cortés et al., 2016), pudimos reconocer la importancia del cuidado como una dimensión relevante de la experiencia de las participantes en torno a los problemas socioambientales identificados durante la primera sesión. A través de diferentes ejercicios participativos - que incluyó un trabajo de reconocimiento de la experiencia ambiental mediante un trabajo con el cuerpo y las emociones la dimensión del cuidado emergió como un eje canalizador de la vivencia, a su vez que agente movilizador de una forma particular de hacer política en la vida cotidiana de las participantes.

En este sentido, observamos resultados similares a los presentados por Sultana (2011) sobre las emociones vivenciadas por mujeres en Bangladesh frente a la contaminación del agua con arsénico. Las mujeres participantes de la Escuela, en este caso, reconocieron el lugar que ocupa el cuidado de la familia y el territorio en sus vivencias cotidianas y en las acciones que toman para enfrentar problemas de contaminación y/o degradación ambiental.

"Se escogió el tema hídrico que la verdad es que a nosotras nos toca mucho como mujeres porque tenemos que preocuparnos de que todo funcione en la casa y en nuestro ambiente. Y eso en base al agua ¿no cierto?” (Dirigenta ade Til Til)

Como usuarias frecuentes del espacio doméstico, donde suelen asumir la responsabilidad de cuidar de hijos, familiares, plantas o animales, generalmente son ellas las que tienden a identificar de manera más inmediata los problemas del entorno, así como a visibilizar alternativas de solución al problema. Con 
relación a este aspecto, resulta especialmente ilustrativo el testimonio de una dirigente de la comuna de Alhué durante la instancia de cierre de la sesión "género y medio ambiente". A partir de su relato, se refleja de qué manera la ética del cuidado de la familia, la comunidad y el territorio cobran importancia al momento de tomar una posición determinada como dirigente social que debe enfrentar el proceso de negociación con una empresa de su localidad que estaba provocando daños en el medioambiente y la salud de los habitantes.

“...uno piensa que lo que está pasando va a afectar a tus hijos, a tu familia, a tus padres, que ya son abuelos, que son ancianos y que no se van a poder ir del lugar o que no se van a querer ir. Y me recordaba una situación en el año 96 cuando se puso una fundidora de baterías camino al sector donde nosotros vivimos [...] Fue puesto además [la fundidora] por un concejal de ese tiempo [...] A mí me resultaba muy raro que solo se trabajara de noche [en la fundidora] y saliera un humo tan oscuro y espeso que era negro, negro [...]. Justamente uno de los trabajadores [de la fundidora] era mi cuñado, y empecé a averiguar qué pasaba. En ese tiempo se estaba armando la primera cooperativa apícola, y para colmo, yo había sido elegida la presidenta de esa cooperativa. Entonces en ese discurso inicial de esa partida de esa cooperativa apícola [...] yo dije que me parecía muy extraño que estuviéramos haciendo la partida de una comunidad apícola, que se suponía que íbamos a producir miel, pero que por otro lado nos estaban metiendo una empresa que nos estaba contaminando. [...] Pero él (concejal que asistió al discurso inicial), como administrador de la empresa [...] lo que hacían era venderle a nuestra comunidad que solo estaban ensamblando vehículos y nos ofrecían cuarenta cupos de trabajo estable para la comunidad con tal de que no los molestáramos. Fue tanto el revuelo del discurso de cómo nos adheríamos como apicultores [...] pero en un mes pudimos desbancar todo y se terminó esa iniciativa. Claro que de por medio me llevé [el reproche] de varios [otros dirigentes de la comunidad] [...] Estuvieron años enojados conmigo porque decían 'pero como estás quitando trabajo para nosotros en el sector [...] que es donde menos tenemos pega'. Y yo siempre me recuerdo un viejito que era papá de una guagüita de seis meses y yo le decía, 'pero vecino, ¿usted no piensa en el futuro de esa guagüita?' (Dirigenta, Comuna de Alhué)

En este caso, la dirigente tuvo que tomar decisiones como presidenta de la cooperativa apícola de su sector, no solo tomando en cuenta a la empresa que les estaba ofreciendo puestos de trabajo a la comunidad, sino que también negociando con otros dirigentes sociales (hombres) de Alhúe que ejercían la dirigencia y tomaban sus decisiones desde otra posición de género. Se puede interpretar, en este caso, que estos dirigentes ejercen una forma de liderazgo masculino que toma con mayor consideración la dimensión productiva (oportunidades laborales) en desmedro de la esfera reproductiva (el cuidado de la familia) al momento de evaluar las 
cargas y beneficios de la instalación de la empresa fundidora de baterías.

Otros resultados similares, como las experiencias feminizadas de sufrimiento ambiental descritas por Arriagada (2019), nos permite reforzar la importancia del cuidado como elemento de agencia política de las mujeres. Esta "conciencia femenina", que deriva de los roles tradicionales de género, además se suele traducir en demandas por compensación en términos de salud, por acceder a mayor bienestar para las familias, mejoramiento del entorno, entre otros. Estos hallazgos también encuentran puntos de convergencia con movimientos de mujeres que, aunque desde una oposición más clara a las relaciones desiguales de género, han levantado una propuesta política en torno a la ética del cuidado (Bolados, 2017).

Desde el enfoque multiescalar que provee la ecología política feminista es posible reconocer, en este sentido, que la manera de participar que tienen las mujeres de la Escuela redescubre y se apropia de una forma de hacer política donde hay una continuidad entre lo privado y lo público, en tanto se reconocen como cuidadoras de los hogares, a su vez que cuidadoras de las comunidades y de sus territorios. Se trata, por tanto, de formas cotidianas y feminizadas de hacer política, que su vez que se constituyen como dispositivos de organización y movilización social en las regiones de estudio.

\section{DISCUSIÓN Y CONCLUSIONES}

Tal como señalamos al inicio de este artículo, el período en que se gestó y desarrolló la Escuela, ocurrió en paralelo a las movilizaciones feministas que remecieron al país durante el primer semestre 2018. Resulta relevante mencionar este acontecer histórico ya que no nos sería indiferente en el marco de la experiencia de realización de la Escuela. Es más, nos atreveríamos a decir que en las ideas, reflexiones, discusiones y eventos que trajo la ola feminista, se jugó parte importante de nuestra apertura hacia nuevas posiciones epistemológicas y prácticas políticointelectuales. Durante ese periodo nos planteamos preguntas tales como: ¿qué rol cumplimos en las distintas esferas de nuestra vida social como mujeres? ¿Cuáles de nuestras prácticas cotidianas han contribuido a reproducir lógicas coloniales o patriarcales de conocimiento? ¿Cómo pensar una forma de ser/pensar/actuar en el mundo, que represente una alternativa a esas formas de dominación? Y, particularmente ¿Cómo nos pensamos como dirigentes/investigadoras en torno a los conflictos ambientales actuales? Para responder a estas preguntas, nos aventuramos a un ejercicio de experimentación con nuestros colaboradores de la FUSUPO, COES y varias dirigentas de las comunas rurales de la Región Metropolitana, para explorar conjuntamente otras formas de construir conocimiento sobre la relación entre género y medioambiente. En esta aventura fue crucial contar con la flexibilidad suficiente como para explorar alrededor de esas 

experiencia de investigación-acción participativa con mujeres dirigentes. Revista de Sociología, 34(1), 147-165. doi: 10.5354/0719-529X.2019.54270

otras posibles respuestas que no necesariamente fuesen las planteadas de manera explícita desde el feminismo académico. Partimos esta iniciativa con un doble propósito: comprender las experiencias y formas de acción diferenciadas por género en torno a las problemáticas ambientales actuales y apostar por una forma de construcción de conocimiento más horizontal entre academia, instituciones y comunidades.

El espectro amplio de elementos teóricos que nos brinda la Ecología Política Feminista, desde sus reflexiones en torno la producción de conocimiento ambiental situado, la posibilidad de navegar entre escalas de análisis, el foco de estudio en los cuerpos de las personas afectadas por ambientes degradados o el reconocimiento de experiencias cotidianas de género que llevan a otras formas de hacer dirigencia y movilizarse, nos permitió superar parte de las contradicciones entre teoría y práctica. Esto, a su vez, se fortaleció gracias a la apertura epistemológica y metodológica de la IAP, cuyos principios nos obligaban a repensar de manera constante los objetivos de cada sesión, las temáticas abordadas, los tiempos de trabajo y los actores externos invitados a participar.

Tomando estos principios teóricos, epistemológicos y metodológicos, hemos querido ilustrar parte de las ideas que dieron origen a la iniciativa Escuela de Dirigentas en temáticas socioambientales y territoriales. A su vez, hemos querido mostrar como esas ideas se expresaron y materializaron - o se pusieron en cuestión durante las sesiones de la Escuela. En este artículo hemos puesto el acento en el entrecruce entre Ecología Política Feminista e IAP para comprender, en primer lugar, como las mujeres rurales de la Región Metropolitana experimentan las intervenciones y transformaciones ambientales en sus territorios. En segundo lugar, para plantear alternativas de producción de conocimiento para la acción ambiental de forma más horizontal y participativa.

Como señalaría Sultana (2011), el lazo se va formando no solo a partir de las luchas socioambientales sino por la similitud de las experiencias y las narrativas compartidas. Para nosotras, el ejercicio de reconocernos como mujer dirigente, mujer investigadora, o mujer trabajadora, así como nuestro rol en las problemáticas socioambientales del Chile contemporáneo, constituye un punto de partida para examinar el rol de académicos y académicas en las luchas ambientales actuales. En este sentido, este artículo también se constituye como un llamado a propiciar la proliferación de espacios de experimentación, donde converjan la investigación y la acción, relevando además la perspectiva de género. Esto permitirá abrir espacios para seguir reflexionando en torno a la producción de conocimiento socioambiental en general, al proceso enseñanza-aprendizaje en la academia, además de ensayar formas de acompañamiento a las organizaciones y movimientos sociales que permitan no solo desafiar las construcciones académicas, sino fortalecer el involucramiento de investigadores e investigadoras en la transformación social. 


\section{REFERENCIAS}

Agarwal, B. (1992). The gender and the environment debate: Lessons from India. Feminist Studies, 18(1), 119-158. doi: $10.2307 / 3178217$.

Alimonda, H. (2006). Una nueva herencia en Comala. Apuntes sobre la ecología política latinoamericana y la tradición marxista. En H. Alimonda (Ed.), Los tormentos de la materia. Aportes para una ecología política latinoamericana (pp. 93-121). Buenos Aires: CLACSO.

Alimonda, H. (2011). La colonialidad de la naturaleza. Una aproximación a la ecología política latinoamericana. En H. Alimonda (Ed.), Naturaleza colonizada. Ecología política y minería en América Latina (pp. 2158). Buenos Aires: CLACSO.

Arevalo, A., Fernández, B., Hidalgo, F., Lepe, Y., Miranda, C., Núñez, M. \& Reyes, L. (2016). Corporalidades y narrativas docentes: Un dispositivo metodológico para la investigación y formación de profesores. Estudios Pedagógicos, 17(4), 223-242. doi: 10.4067/S0718-07052016000500013.

Arriagada, E. (2019). Las "madres del plomo": Women's environmental activism and suffering in northern Chile. En A. Ramm \& J. Gideon (Eds.), Motherhood, social policies and women's activism in Latin America. Palgrave Macmillan (forthcoming).

Auyero, J. \& Swistun, D. (2008). Inflamable. Buenos Aires: Paidós.

Bell, S. \& Braun, Y. A. (2010). Coal, identity, and the gendering of environmental justice activism in central Appalachia. Gender \& Society, 24(6), 794-813. doi: 25789907.

Bolados, P. (2017). Una ecología política feminista en construcción: El caso de las "Mujeres de zonas de sacrificio en resistencia", Región de Valparaíso, Chile. Psicoperspectivas, 16(2), 33 - 42. doi:
10.5027/psicoperspectivas-Vol16-Issue2fulltext-977

Buechler, S. \& Hanson A. M. (2015). A Political Ecology of Women, Water and Global Environmental Change. New York: Routledge.

Bustos, B., Prieto, M. \& Barton, J. (2015). Ecología política en Chile: Naturaleza, propiedad, conocimiento y poder. Santiago, Chile: Editorial Universitaria.

Butler, J. (1999). Gender trouble: feminism and the subversion of identity. New York: Routledge.

Carney, J. (1992). Peasant women and economic transformation in the Gambia. Development and Change, 23(2), 67-90. doi: 10.1111/j.1467-7660.1992.tb00446.x

Cortés, X., Díaz, N., Flores, V., \& Monsalves, S. (2016, Noviembre 16-18). La experiencia corporizada en los procesos de enseñanzaaprendizaje de metodologías de Investigación Acción Participativa. Ponencia presentada en $\mathrm{V}$ Encuentro Latinoamericano de Metodología de las Ciencias Sociales. Mendoza, Argentina.

Elmhirst, R. (2018). Ecologías políticas feministas: Perspectivas situadas y abordajes emergentes. Ecología Política, 54(1), 52-59. doi: 10.1080/03066150.2017.1337002

Escobar, A. (2011). Ecología política de la globalidad y la diferencia. En H. Alimonda (Ed.), Naturaleza colonizada. Ecología política y minería en América Latina (pp. 6192). Buenos Aires: CLACSO.

Fals-Borda, O. (2009). Una sociología sentipensante para América Latina. Bogotá: Siglo del Hombre Editores / CLACSO.

Güereca, R. (2016). Claves para una sociología feminista. En N. Blázquez \& M. Castañeda (Coords.), Lecturas críticas en investigación 
Zambra Álvarez, A., \& Arriagada Oyarzún, E. (2019). Género y conflictos socioambientales: Una experiencia de investigación-acción participativa con mujeres dirigentes. Revista de Sociología, 34(1), 147-165. doi: 10.5354/0719-529X.2019.54270

feminista (pp. 91-123). Ciudad de México: Universidad Autónoma de México.

Haraway, D. (1991). Simians, cyborgs and women: the reinvention of nature. New York: Routledge.

Harding, S. (1986). The science question of feminism. New York: Cornell University Press.

Harris, L. (2006). Irrigation, gender, and social geographies of the changing waterscapes of Southeastern Anatolia. Environment and Planning, 24(2), 187-213. doi: 10.1068/d03k.

Harris, L. (2015). Foreword A quarter century of knowledge and change: pushing feminism, politics, and ecology in new directions with feminist political ecology. En S. Buechler \& A.M. Hanson (Eds.), A political ecology of women, water and global environmental change (pp. 19-24). New York: Routledge.

Harris, L. (2017). Theorizing gender, ethnic difference and inequality in relation to water access and politics in southeastern Turkey. En C. Ashcraft \& T. Mayer (Eds.), The politics of freshwater: Access, conflict and identity (pp. 141-155). New York: Routledge.

Hawkins, R., Ojeda, D., Asher, K., Baptise, B., Harris, L., Mellott, S., Nightingale, A., ... Sultana, F. (2011). A discussion: Gender and environment: Critical tradition and new challenges. Environmment and Planning D: Society and Space, 29(2), 237-253. doi: 10.1068/d16810.

Kaplan, T. (1997). Crazy for democracy: Women in grassroots movements. New York $\mathrm{y}$ London: Routledge.

Kennedy, E. H. \& Dzialo, L. (2015). Locating gender in environmental sociology. Sociology Compass, 9 (10), 920-929. doi: $10.1111 /$ soc 4.12303 .

Krauss, C. (2008). Challenging power. Toxic waste protest and the politicization of white, working-class women. En N. Naples (Ed.), Community activism and feminist politics. Organizing across race, class, and gender (pp. 129-150). New York y London: Routledge.

Leff, E. (2006). La ecología política en América Latina. Un campo en construcción. En H. Alimonda (Ed), Los tormentos de la materia. Aportes para una ecología política latinoamericana (pp. 21-39). Buenos Aires: CLACSO.

Merchant, C. (1980). The death of nature: Women, ecology and the scientific revolution. San Francisco, CA: Harper Row.

Nightingale, A. (2006). The nature of gender: Work, gender and environment. Environment and Planning D: Society and Space, 24(2), 165-185. doi: 10.1068/d01k

Ojeda, D. (2011). Género, naturaleza y política: Los estudios sobre género y medio ambiente. Halac Belo Horizonte, 1(1), 55 - 73.

Plumwood, V. (1993). Feminism and the matery of nature. Londres: Routledge

Red CIMAS (2009). Metodologías Participativas: Manual. Madrid: Autor.

Robbins, P. (2012). Political ecology: A critical introduction. Chichester, Reino Unido: Wiley \& Sons.

Rocheleau, D., Thomas-Slayter, B., \& Wangari, E. (2004). Género y medio ambiente: Una perspectiva de la ecología política feminista. En V. Vásquez \& M. Velásquez (Eds.), Miradas al futuro: hacia la construcción de sociedades sustentables con equidad de género (pp. 343-372). México: Centro Internacional de Investigaciones para el Desarrollo.

Servicio País. (2019). Servicio País Fundación Superación Pobreza. Santiago, Chile. Recuperado de: http://www.serviciopais.cl/conocenos/

Shiva, V. \& Mies, M. (1993). Ecofeminism. Londres: Zed Books.

Sultana, F. (2011). Suffering for water, suffering from water: Emotional geographies of resource, access, control and conflict. Geoforum, 42(2), 163 - 172. doi: 10.1016/j.geoforum.2010.12.002. 


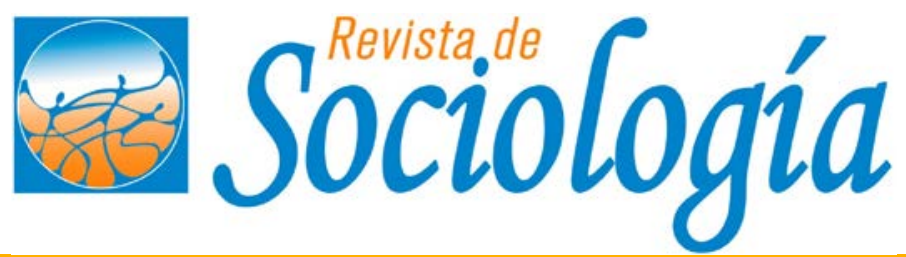

Sundberg, J. (2017). Feminist political ecology. En D. Richardson, N. Castree M.F. Goodchild, A. Kobayashi, W. Liu \& R. Marston (Eds.), International encyclopedia of geography: People, the earth,
Zambra Álvarez, A., \& Arriagada Oyarzún, E. (2019). Género y conflictos socioambientales: Una experiencia de investigación-acción participativa con mujeres dirigentes. Revista de Sociología, 34(1), 147-165. doi: 10.5354/0719-529X.2019.54270

environment and technology. Chichester: Reino Unido: Willey \& Sons.

Manuscrito recibido: 30-05-2019

Manuscrito aceptado: 05-08-2019 